DBA/1 mice were immunized by intradermal injection at day-0 and at day-21 with a grade chick type II collagen and complexe Freund's adjuvant. Monitoring of arthritis indices was done from day-25 to day-35. At day-35, monocytes were isolated from bone marrow by negative selection. To generate MDMs, purified monocytes were incubated 6 days in the presence of M-CSF+IL-4 (M2-like). Expressions of total macrophages markers (CD11b and F4/80), M2-like macrophage polarization markers (CD206, IL-10 and Arg-1) were evaluated by flow cytometry, ELISA or qPCR.

Results: Overexpression of miR-155 in HD monocytes significantly decreased the gMFI of the pan-macrophages markers, CD11b and CD71, but also the M2-like markers, CD206 and CD163 after 6 days of culture with human SAB. Likewise, we demonstrated a significant reduction of Arg-1 expression and increase in iNOS expression in M2-like macrophages after miR-155 transfection of HD monocytes (Fig. 1A).

Conversely, ex vivo silencing of miR-155 with antagomir in RA monocytes restored the defect of $\mathrm{M} 2$-like phenotype macrophages by increasing the percentage of pan-macrophages markers, CD11b and CD71 and M2-like macrophages markers CD206 and CD163 after a 6-day culture in human SAB. Moreover, miR-155 antagomir increased significantly the Arg-1 expression and reduced iNOS expression in M2-like macrophages (Fig. 1B).

The defect of monocytes capacity of differentiation into M2-like phenotype was also found in in CIA mice as compared to controls, with a decreased expression of CD206, IL-10 secretion and Arginase-1 expression (Fig. 1C). MiR155 was also involved in this defect in mice: its expression level was the same in M1-like macrophages between controls and CIA mice, whereas it was increased in M2-like CIA macrophages compared to controls mice (Fig. 1C).

Conclusion: MiR155 plays a key role in the specific defect of anti-inflammatory M2 macrophages polarization found both in human rheumatoid arthritis and in CIA mice. MiR155 could represent a new therapeutic target in RA that can be tested in the mouse model of CIA

Disclosure of Interests: Audrey Paoletti: None declared, franceline reynaud: None declared, julien rohmer: None declared, Juliette Pascaud: None declared, Bineta Ly: None declared, Elodie Rivière: None declared, Nicolas Tsapis: None declared, Elias Fattal: None declared, Gaetane Nocturne: None declared, Xavier Mariette Grant/research support from: Servier, Consultant for: AstraZeneca, Bristol-Myers Squibb, GlaxoSmithKline, Janssen, Pfizer, UCB Pharma

DOI: 10.1136/annrheumdis-2019-eular.5415

\section{SAT0058 DEFICIENT DNA DAMAGE RESPONSE AND REPAIR IN ACTIVE RHEUMATOID ARTHRITIS AND THE EFFECT OF TREATMENT}

Maria Pappa ${ }^{1}$, Nikolaos Vlachogiannis ${ }^{1}$, Alexandra Argyriou ${ }^{1}$, Vassilis L. Souliotis ${ }^{1,2}$, Petros Sfikakis ${ }^{1} .{ }^{1}$ First Department of Propaedeutic Internal Medicine and Joint Rheumatology Program, National and Kapodistrian University of Athens, Medical School, Athens, Greece; ${ }^{2}$ Institute of Biology, Medicinal Chemistry and Biotechnology, National Hellenic Research Foundation, Athens, Greece

Background: Increased endogenous DNA damage poses a serious threat to cell health, since it may lead to mutagenesis, genomic instability and cellular apoptosis. DNA damage accumulation in immune cells occurs in patients with chronic inflammatory diseases, however; little is known on the types and prevalence of endogenous DNA damage in patients with Rheumatoid arthritis (RA).

Objectives: To study DNA damage response and repair network in patients with active RA and to test the hypothesis that anti-rheumatic treatment influences this network.

Methods: Peripheral blood mononuclear cells were isolated from 15 patients with active RA and 65 apparently healthy controls; 8 patients were re-examined after 12-week treatment. Endogenous DNA damage was determined using alkaline comet assay. Specific markers for singlestrand DNA breaks (SSBs; RPA32) and double-strand DNA breaks (DSBs; $\gamma \mathrm{H} 2 \mathrm{Ax}$, 53BP1) were measured using western-blot. Formation of DNA damage was assessed by oxidative stress and abasic DNA sites measurements. Chromatin organization and the two subpathways of the fundamental nucleotide excision repair mechanism, namely, transcriptioncoupled repair (TCR) and global genome repair (GGR), were assessed along the $\mathrm{N}$-ras gene.
Results: A 3-fold increase of endogenous DNA damage levels (Olive Tail Moment reflecting both SSBs and DSBs) was evident in active RA [mean $\pm \mathrm{SD} ; 12.8 \pm 7.5$ versus $4.5 \pm 2.4, \mathrm{p}<0.001$ ), as well as induction of RPA32, $\gamma \mathrm{H} 2 \mathrm{Ax}$ and 53BP1, higher oxidative stress levels and increased abasic sites, compared to controls. While TCR capacity was preserved GGR capacity was deficient in all active RA patients. Moreover, a more condensed chromatin structure was found in active RA compared to controls. Following treatment, chromatin structure loosened, GGR capacity was restored, oxidative stress and abasic sites decreased and levels of endogenous DNA damage reached control values in all patients.

Conclusion: Deregulated chromatin organization, deficient DNA repair capacity and augmented formation of DNA damage contribute to the accumulation of endogenous DNA damage in patients with active RA and are reversible after treatment. Additional studies to better understand the negative impact of DNA endogenous damage accumulation may create new therapeutic opportunities for patients with RA

Disclosure of Interests: None declared

DOI: 10.1136/annrheumdis-2019-eular.5129

\section{SAT0059 APOLIPOPROTEIN B DERIVED PEPTIDES INHIBIT PRODUCTION OF PRO-INFLAMMATORY CYTOKINES IN PBMC OF RHEUMATOID ARTHRITIS (RA) PATIENTS AND IMPROVE ARTHRITIS IN ANIMAL MODEL OF RA}

Ji Soo Park ${ }^{1,2}$, Joo Youn Lee ${ }^{2}$, Hyun Jung Yoo ${ }^{2}$, Jeong Yeon Kim ${ }^{1,2}$, Ji Hye Lee ${ }^{2}$, Seon Uk Kim ${ }^{1,2}$, Ra Ham Kim ${ }^{1,2}$, Jin Kyun Park ${ }^{2}$, Eun Young Lee ${ }^{2}$, Eun Bong Lee ${ }^{2}$ Eugene C. Yi ${ }^{1}$, Yeong Wook Song ${ }^{1,2} .{ }^{1}$ Department of Molecular Medicine and Biopharmaceutical Sciences, Graduate School of Convergence Science and Technology, and College of Medicine, Seoul National University, Seoul, Korea, Rep. of (South Korea); ${ }^{2}$ Division of Rheumatology, Department of Internal Medicine, College of Medicine, Seoul National University Hospital, Seoul, Korea, Rep. of (South Korea)

Background: Alpha-enolase (ENO1) is a multifunctional glycolytic enzyme The cell-surface expression of ENO1 is increased in monocytes isolated from peripheral blood mononuclear cells (PBMCs) of RA patients. In previous experiments, we reported apolipoprotein $B(a p o B)$ to be as a novel ligand of cell surface expressed ENO1 and to enhance chronic inflammation in RA (1)

Objectives: This study was performed to evaluate agonistic or antagonis tic function of peptide sequences within apoB, which modulated severity of arthritis in K/BxN serum transfer arthritis mouse model.

Methods: Peptides binding to ENO1 were evaluated by peptide microarray. Peptides were designed to have twelve amino acids lengths with ten amino acids overlap. Levels of pro-inflammatory cytokines produced by PBMCs were measured after treatment with peptides in RA patients and healthy controls (HCs). To evaluate the antagonistic function of peptides RA PBMCs pre-treated with peptides were stimulated with apoB and proinflammatory cytokines were measured in culture supernatant. Peptides were injected in $\mathrm{K} / \mathrm{BxN}$ serum transfer mice and we measured the thickness of ankles and arthritis score to determine whether peptides could reduce arthritis severity.

Results: Among eighty five peptides from apoB, five peptides exhibited strong binding to ENO1. Two peptides increased production of pro-inflam matory cytokines, IL-1 $\beta$, IL- 6 and TNF- $\alpha$, but three peptides did not change levels of pro-inflammatory cytokines in RA and HC PBMCs. How ever the three peptides reduced production of pro-inflammatory cytokines in RA PBMCs stimulated with apoB. These three peptides were named IP (inhibitory peptide)1, IP2 and IP3. IPs were injected after induction of the arthritis in $\mathrm{K} / \mathrm{BxN}$ serum transfer mice in vivo. Ankle thickness and arthritis scores were substantially reduced in IPs treated group than control group, and synovial inflammation, bone erosion and cartilage damage of arthritis were less severe in the IPs-treated groups. Especially, IP2 showed the most significant effect.

Conclusion: The apoB derived peptides interacting with ENO1 could have novel therapeutic effect in RA.

\section{REFERENCE}

[1] Lee, J. Y., et al. (2018). "Apolipoprotein B binds to enolase-1 and aggravates inflammation in rheumatoid arthritis." Ann Rheum Dis 77(10): 1480 1489

Disclosure of Interests: None declared DOI: 10.1136/annrheumdis-2019-eular.6458 